\title{
Roboter zur Unterstützung im Alter
}

\author{
Esther Ruf (D) - Stephanie Lehmann $(\mathbb{D} \cdot$ Cora Pauli $\cdot$ Sabina Misoch
}

Eingegangen: 30. Juni 2020 / Angenommen: 13. Oktober 2020 / Online publiziert: 3. November 2020

(C) Der/die Autor(en) 2020

Zusammenfassung Wir begegnen unterschiedlichen gesellschaftlichen Herausforderungen, wie steigendem Anteil der älteren Bevölkerung, Fachkräftemangel im Pflegesektor und Wunsch älterer Personen möglichst lange selbständig in ihrer gewohnten Häuslichkeit zu leben. Eine Möglichkeit auf diese Entwicklungen zu reagieren, ist Roboter zur Unterstützung im Alter einzusetzen. Vielfältige Auswirkungen eines Robotereinsatzes wurden in der Literatur beschrieben und in der gerontologischen Forschung diskutiert. Um jedoch die intendierte Wirkung zu entfalten, müssen die Roboterlösungen akzeptiert werden. Die Akzeptanzbedingungen erforscht das Institut für Altersforschung (IAF) der OST - Ostschweizer Fachhochschule, unter partizipativem Einbezug der Nutzenden. Anhand von Ergebnissen aus Befragungen und Forschungsprojekten stellt dieser Artikel die Erfahrungen von Personen mit und die Einstellungen gegenüber Robotern vor. Faktoren der Roboterakzeptanz werden allgemein und mit dem Fokus auf Emotionen gegenüber Robotern und bevorzugtes Aussehen eines Roboters aufgegriffen. Exemplarisch werden für die Bereiche Privathaushalt und Alters- und Pflegeheim Einsatzmöglichkeiten für Roboter zur Unterstützung im Alter vorgestellt. Es werden aber auch Grenzen und Schwierigkeiten eines Robotereinsatzes in Bezug auf technische Machbarkeit, gewünschte Funktionen, und ethischen Überlegungen aufgezeigt. Es wird klar, dass das Thema interdisziplinär angegangen werden muss, um gemeinsam die beste Lösung für Nutzergruppen und Gesellschaft zu erlangen. Neben finanziellen Überlegungen und einer systematischen Analyse des Mehrwerts ist der frühe Einbezug aller Endnutzenden notwendig. So kann die Akzeptanz einzelner Roboteranwendungen sichergestellt, Verbesserungspotential früh erkannt und Roboter erfolgreich eingesetzt werden.

E. Ruf $(\bowtie) \cdot S$. Lehmann $\cdot$ C. Pauli $\cdot$ S. Misoch Institut für Altersforschung, OST - Ostschweizer Fachhochschule, St. Gallen, Schweiz E-Mail: esther.ruf@ost.ch 
Schlüsselwörter Serviceroboter · Akzeptanzmodelle $\cdot$ Roboterakzeptanz ·

Senioren/innen · Partizipation · Emotionen · Privathaushalt · Alters- und Pflegeheim

\section{Robots to support older adults}

Abstract Our society is confronted with various challenges such as the increase in the older population group, the shortage of skilled workers in the care sector and the desire of older adults to live independently in their homes as long as possible. One possibility to meet these challenges is the use of robots to support older adults. Various effects of the use of robots have been described in the literature and discussed in gerontological research. However, to achieve the intended effect, the robotic solutions must be accepted. The Institute for Ageing Research (IAF) of the Eastern Switzerland University of Applied Sciences (OST) conducts research regarding the acceptance with participatory involvement of the users. Based on results from surveys and research projects, this article shows the experiences of people with robots and their attitudes towards them. Factors of robot acceptance are discussed in general terms and with a focus on emotions towards robots and preferred appearances of robots. Examples of applications for robots in the areas of the private household and nursing homes are presented. Limitations and difficulties of robot use in terms of technical feasibility, desired functions, and ethical considerations are also shown. It becomes clear that an interdisciplinary approach to the topic is essential to achieve the best solution for user groups and society. Besides financial considerations and a systematic analysis of the added value, the early involvement of all end users is necessary. In this way, the acceptance of individual robot applications can be ensured, potential for improvement can be identified early on and robots can be used successfully.

Keywords Service robots - Acceptance models · Robot acceptance - Older adults · Participation · Emotions · Private home · Nursing home

\section{Einleitung}

Wie in allen Industrieländern (Vaupel 2000) steigt auch in der Schweiz der Anteil der Personen, die 65 Jahre und älter sind, stetig an. So betrug im Jahr 2019 der Anteil der über 65-Jährigen 18,7\% (Bundesamt für Statistik [BFS] 2020a). Dieser Anteil wird gemäss Referenzszenario des Bundes auf mindestens 25,6\% im Jahr 2050 ansteigen (BFS 2020b), aufgrund verschiedener zugrundeliegender Entwicklungen, wie einer niedrigen Geburtenrate und einer steigenden Lebenserwartung. Viele Personen altern bei guter Gesundheit, jedoch nehmen mit zunehmendem Alter funktionelle Einschränkungen und Erkrankungen zu (BFS 2019). Daher sind die meisten Personen irgendwann auf Unterstützung und/oder Pflege angewiesen. Zudem besteht ein Fachkräftemangel im Pflegesektor, welcher sich in den nächsten Jahren weiter verstärken wird (Mercay und Grünig 2016; World Health Organization 2015), sowohl im stationären Bereich der Alters- und Pflegeeinrichtungen als auch im ambulanten häuslichen Bereich. Gleichzeitig möchte eine Mehrheit der älteren 
Personen möglichst lange selbständig und autonom leben und in ihrer gewohnten Häuslichkeit verbleiben (Hedtke-Becker et al. 2012).

Eine Möglichkeit den gesellschaftlichen Herausforderungen wie Fachkräftemangel und steigenden Gesundheitskosten als auch den Wünschen der älteren Bevölkerungsgruppe nach Autonomie und Selbstbestimmung zu begegnen, wird darin gesehen, Roboter im Bereich des Wohnens und Lebens älterer Menschen einzusetzen (Kehl 2018; Robinson et al. 2014; Wu et al. 2014).

Der Begriff des Roboters wird unterschiedlich definiert (Decker 2010), wobei sich die Definitionen meist nur in Details unterscheiden, je nach definierender Fachrichtung, Vereinigung oder Fokussierung auf technische oder funktionale Attribute. Aufgrund ihrer Anwendung können Roboter eingeteilt werden in Industrieroboter (Roboter für die Fertigung) und Serviceroboter (Roboter für Dienstleistungen). Bei Servicerobotern als eigene Unterklasse von Robotern steht der direkte Nutzen für den Menschen im Vordergrund. Die internationale Organisation für Standardisierung definiert einen Serviceroboter als Roboter ,that performs useful tasks for humans or equipment excluding industrial automation applications (ISO 8373)“ (International Federation of Robotics 2020).

Das Angebot an Servicerobotern zur Unterstützung von Menschen wird in der Literatur jeweils unterschiedlich eingeteilt. Becker et al. (2013) gliedern Roboter in Betreuung und Gesundheitsversorgung anhand der Anwendung in:

- Trainingsgeräte und Hilfsmittel zur Bewegungsausführung, Mobilität und Selbständigkeit, welche den Menschen darin unterstützen, bestimmte Bewegungen und Handlungen zu trainieren oder auszuführen,

- Telepräsenz- und Assistenzroboter, welche die Anwesenheit eines Menschen, zum Beispiel einer Pflegekraft, eines Arztes oder eines Therapeuten, ersetzen oder eine Person in der Ausführung von Handlungen unterstützen, und

- Sozial-interaktive Roboter, welche vor allem das Ziel haben, mit Menschen zu interagieren und ihnen als Begleiter oder Gefährten zu dienen.

Eine weitere weit verbreitete Unterteilung ist diejenige von Broekens et al. (2009), welche unterstützende Roboter für Ältere einteilen in Rehabilitationsroboter und Sozial Assistive Roboter, mit den Unterkategorien Serviceroboter und Companion Roboter (S. $94 \mathrm{ff}$.).

Obwohl Roboter in Schweizer Institutionen für Menschen im Alter aktuell noch wenig verbreitet sind, wird in der gerontologischen Forschung der Einsatz von Robotern viel diskutiert (Seifert und Ackermann 2020). Im vorliegenden Beitrag liegt der Fokus auf Servicerobotern im Altersbereich, welche verschiedene Aufgaben übernehmen können, mit dem Ziel, Aktivitäten des täglichen Lebens zu unterstützen oder dazu anzuregen, soziale Interaktion und Kommunikation zu ermöglichen, sowie pflegerische Tätigkeiten zu unterstützen.

In der Literatur werden vielfältige mögliche positive Auswirkungen eines Robotereinsatzes beschrieben. So könnten ältere Personen durch den Einsatz von Robotern zuhause von gesteigerter Autonomie, Mobilität und Sicherheit profitieren, indem ihre Unabhängigkeit, ihr Wohlbefinden und ihre Lebensqualität positiv beeinflusst werden (Becker et al. 2013; Sixsmith und Gutmann 2013; Wu et al. 2014). In ihrem Review berichten Agnihotri und Gaur (2016) über vielversprechende An- 
wendungen von Assistenzrobotern für ältere Personen im Gesundheitsbereich. Bezüglich Sozial Assistiver Roboter zeigten Abdi et al. (2018) in ihrem Review fünf mögliche Einsatzbereiche auf und fanden überwiegend positive Befunde bei deren Einsatz. Für pflegende Angehörige und Pflegefachpersonen könnten sich Belastungen durch robotische Systeme im ambulanten und stationären Bereich reduzieren, indem Roboter bei körperlich anstrengenden, pflegefernen und Routine-Aufgaben unterstützen. Dies bietet auch zeitliche und psychische Entlastung (Becker und Khiri 2018; Merda et al. 2017). Diese Ergebnisse basieren jedoch auf experimentellen und Beobachtungsstudien sowie Feldtests und können daher nicht verallgemeinert werden. Abgesehen von einzelnen positiven Berichten gibt es bisher wenig belastbare Studien in größeren Populationen, welche diese Ergebnisse unterstützen. Aus pflegewissenschaftlicher Perspektive resümieren Hülsken-Giesler und Daxberger (2018), dass der Erkenntnisstand bezüglich der Rahmenbedingungen und den Effekten eines Einsatzes von Robotern in der Pflege noch unzureichend ist und weisen darauf hin, dass die besonderen Charakteristika des professionellen pflegerischen Handelns als personenbezogene Dienstleistung bisher kaum angemessen berücksichtigt werden. Vor allem die spezifischen Bedürfnisse unterschiedlicher pflegerischer Handlungsfelder, wie stationäre Akutpflege, stationäre Langzeitpflege, und ambulante Pflege müssen dabei berücksichtigt werden.

Damit neue technische Entwicklungen wie Roboter eingesetzt werden und ihre intendierte Wirkung entfalten können, müssen sie von den Endnutzenden, den Senioren/innen und Pflegefachpersonen, akzeptiert werden. Partizipative Methoden erleichtern die Entwicklung nachhaltiger Roboteranwendungen (Lee et al. 2017), besonders die frühe partizipative Einbindung der Endnutzenden ist dabei für die Akzeptanz essentiell (Lehmann et al. 2017), auch wegen der stark emotionalen Besetzung des Themas (Merda et al. 2017).

Das Institut für Altersforschung (IAF) der OST - Ostschweizer Fachhochschule, erforscht die Akzeptanzbedingungen für einen Robotereinsatz bei älteren Personen mit Fokus auf dem partizipativen Einbezug der Nutzenden (Lehmann et al. 2017) in einem möglichst frühen Stadium der Roboterentwicklung anhand unterschiedlicher Forschungsmethoden. Die Fokussierung auf die Sichtweise der Endnutzenden erlaubt es, förderliche und hinderliche Faktoren aufzudecken und durch deren Berücksichtigung den Nutzen sicherzustellen, die Akzeptanz zu erhöhen, und damit das Produkt für den Markt erfolgreicher zu machen.

Dieser Artikel zeigt exemplarisch Einsatzmöglichkeiten für Roboter zur Unterstützung im Alter anhand von Ergebnissen aus Befragungen und Forschungsprojekten, sowie Schwierigkeiten und die Wirkung eines Einsatzes auf. Dabei werden Ergebnisse berichtet, die anhand verschiedener Methoden erhoben wurden. Dabei ist zu beachten, dass quantitative Methoden sehr hohe Teilnehmerzahlen benötigen, um valide Ergebnisse zu erzielen, und aktuell im Bereich der Robotik im Altersbereich (u.a. wegen Verfügbarkeit und hoher Kosten der Roboter) aufwändig anzuwenden sind. Daher ist es wichtig ebenso qualitative Methoden und Living Lab-Ansätze einzusetzen, um in der frühen Phase der Technikentwicklung für Technikaneignung und Akzeptanz valide Ergebnisse zu erhalten (Abowd et al. 2002; Chi 2009; Mayring 2000). 


\section{Erfahrungen und Einstellung gegenüber Robotern}

Die meisten Personen haben bis dato noch keine direkten Erfahrungen ${ }^{1}$ mit Robotern gemacht. In einer repräsentativen telefonischen Befragung $(N=1003)$ zum Thema „Service-Robotik: Mensch-Technik-Interaktion im Alltag“ in Deutschland gaben $26 \%$ (forsa 2016) und in einer Online-Befragung, die 2015 in Japan, den USA und Deutschland zum Thema Roboter und künstliche Intelligenz durchgeführt wurde, $27 \%$ der Personen in Deutschland an ( $N=1382$, Altersrange 16-59 Jahre), bereits mit einem Roboter zu tun gehabt zu haben (Nitto et al. 2017). Im Eurobarometer von 2017, das die öffentliche Meinung in den 28 Mitgliedstaaten zu einer Reihe von Bereichen, die mit digitalen Technologien, Roboter und künstlicher Intelligenz zu tun haben, erhebt, wurden per Zufallsauswahl 27.901 EU-Bürger/innen ab 15 Jahren zu Hause in ihrer Muttersprache face-to-face interviewt (European Commission 2017). $14 \%$ der so befragten Europäer/innen gaben an, bereits Erfahrung mit einem Roboter zu haben.

Bezüglich der generellen Einstellung gegenüber Robotern gibt es widersprüchliche Befunde (Becker 2018). Wu et al. (2014) liessen elf ältere Personen (9 Frauen, 2 Männer; $M=79,3$ Jahre), ohne und mit leichten kognitiven Einschränkungen vier Wochen lang einmal pro Woche mit einem Roboter interagieren. Im robot-acceptance questionnaire und im anschliessenden halbstrukturierten Interview wurden von den Teilnehmenden eine geringe Intention den Roboter zu nutzen und negative Einstellungen dem Roboter gegenüber berichtet. Daten des Eurobarometers von 2014 aus den EU-Mitgliedstaaten ( $N=27.801, \mathrm{M}=50,2 \mathrm{Jahre}, \mathrm{SD}=18,2 ; 55 \%$ Frauen $)$ zeigten, dass es in der Bevölkerung Bedenken bezüglich eines Robotereinsatzes hinsichtlich möglicher negativer Auswirkungen auf ältere Menschen gibt, dabei waren die älteren Befragten ablehnender eingestellt als die jüngeren (Hudson et al. 2017). Arras und Cerqui (2005) befragten während der Schweizer Landesaustellung (Expo.02) im „Robotik“ Pavillon, in welchem zunächst mit elf Robotern interagiert werden konnte, 2042 freiwillige Personen mit einem Papier-Fragebogen auf Deutsch oder Französisch. Auch bei ihnen äusserten sich die älteren Personen eher skeptisch gegenüber neuen Technologien, waren jedoch durchaus bereit diese Skepsis zu überwinden, wenn sie sich positive Effekte auf die Lebensqualität und ihre Unabhängigkeit vorstellen konnten. In der Stichprobe waren ältere Personen über 65 Jahre (11\% über 60 Jahre), Frauen (44\%) und tiefere Bildungsschichten unterrepräsentiert. Ray et al. (2008) hingegen fanden in ihrer Befragung, die aus halbstrukturierten Interviews ( $N=11$, Altersrange 20-57 Jahre) und einem Fragebogen $(N=240,6 \%$ über 65 Jahre) auf Französisch, verteilt an einer Messe in Genf, bestand, eine sehr positive Einstellung gegenüber Robotern und in der repräsentativen telefonischen Befragung in Deutschland konnten sich 56\% grundsätzlich vorstellen, einen Serviceroboter zu kaufen (forsa 2016).

\footnotetext{
1 Die „Erfahrung“ mit einem Roboter wird dadurch erfragt, ob die Person bereits einmal persönlich mit einem Roboter zu tun hatte (forsa 2016) oder konkret in welchem Bereich (Zuhause, bei der Arbeit, oder anderswo) bereits einmal direkt Kontakt mit einem Roboter bestand (European Comission 2017; Lehmann et al. 2020b; Nitto et al. 2017).
} 
Einstellungen der Bevölkerung zu technischen und digitalen Anwendungen in der Pflege wurden zum Beispiel im Rahmen des ZQP-Reports des Zentrum für Qualität in der Pflege (2019) mit computergestützten Telefoninterviews erhoben, basierend auf einer Stichprobe von Privathaushalten in Deutschland ( $N=1000$, ab 18 Jahren). Dabei zeigte sich, dass über die Hälfte der Befragten befürwortet, die Pflege durch einen Roboter zu unterstützen (jeweils mindestens $51 \%$, je nach Einsatzgebiet des Roboters steigt die Befürwortung bis auf 76\%, wie zum Beispiel als Erinnerungshilfe zur Medikamenteneinnahme). Dass viele Personen offen gegenüber digitalen Helfern in der Pflege sind, zeigte eine weitere repräsentative telefonische Befragung ( $N=1004$, ab 18 Jahren) (Bitkom Research 2018). 41\% konnten sich vorstellen, dass ein Roboter sie zumindest teilweise pflegt. Von den 18- bis 29-Jährigen konnten sich dies $51 \%$ und bei den Personen über 65 Jahren $37 \%$ vorstellen. Im Rahmen einer Befragung von professionellen Pflegekräften ( $N=127$, Altersrange 19-71 Jahre) konnten Zöllick et al. (2020) internationale Ergebnisse für den deutschsprachigen Raum bestätigen, indem sie zeigten, dass Pflegekräfte einen Einsatz von Technik für die körperliche Unterstützung der eigenen Tätigkeit als Entlastung sehen und diese zu schätzen wissen, jedoch beim Technikeinsatz zur sozialen und emotionalen Zuwendung starke Vorbehalte haben.

Zusammenfassend ist festzustellen, dass die Akzeptanz von Robotern verschiedener Interessensgruppen sehr unterschiedlich sein kann und die Technologieakzeptanz stark von der Gruppe beeinflusst wird, zu welcher die nutzende Person gehört (Pino et al. 2015).

Um der rasanten Technikentwicklung, der widersprüchlichen Datenlage und der Kultursensitivität bei Einstellungen zu Robotern Rechnung zu tragen, wurde vom IAF eine Online-Befragung mit geschlossenen selbst zusammengestellten Fragen aus bekannten Akzeptanz-Skalen in der Deutschschweiz durchgeführt, welche speziell die Akzeptanz von Robotern zur Unterstützung im Alter erhob (Lehmann et al. 2020b). Von Januar 2019 bis März 2020 nahmen 189 Personen an der Befragung teil (Altersrange 17-96 Jahre, $\mathrm{M}=65,4, \mathrm{SD}=16,6 ; 57,6 \%$ Frauen). 42\% der Befragten dieser Umfrage hatten bereits einmal direkt mit einem Roboter zu tun. Es zeigte sich, dass 67,5\% der Befragten eine positive Sicht von Robotern hatten, und 69,7\% einen Roboter nutzen würden. Diese Nutzung traute sich die Mehrheit auch zu. Bei möglichen Personengruppen, bei denen die Befragten angeben sollten, ob sie sich einen Einsatz vorstellen könnten, zeigte sich, dass bei einem Robotereinsatz für die eigenen Eltern oder Grosseltern nur 20,9\% der Befragten zustimmten.

\section{Faktoren der Roboterakzeptanz}

Es gibt verschiedene Faktoren, welche einen Einfluss auf die Akzeptanz haben, die sich eher der nutzenden Person selbst, der genutzten Technik oder auch den Umweltbedingungen zuordnen lassen (Broadbent et al. 2010) oder eher kognitiv evaluativ, sozial oder emotional zu bewerten sind. Auch die Generationszugehörigkeit hat eine starke Auswirkung auf die Einstellung zu Technik, ihre Nutzung und Bewertung (Misoch et al. 2016). Nach einer kurzen Darstellung der theoretischen Modelle der Technikakzeptanz werden einzelne Faktoren der Akzeptanz, wie Emo- 
tionen der Nutzenden, Aussehen des Roboters und Situation des Robotereinsatzes, aus der Forschung des IAF detaillierter dargestellt.

\subsection{Technikakzeptanzmodelle}

Modelle zur Technikakzeptanz modellieren unterschiedliche Einflüsse auf die Absicht des Nutzers, die Technik zu verwenden, und gehen davon aus, dass die geäusserte Absicht Hinweis auf die tatsächliche Nutzung gibt. Ein Grundmodell ist das Technology Acceptance Model (TAM) (Davis et al. 1989). Dies wurde im Laufe der Zeit um weitere Einflussfaktoren erweitert (TAM 2, Venkatesh und Davis 2000; TAM 3, Venkatesh und Bala 2008; Unified Theory of Acceptance and Use of Technology, UTAUT, Venkatesh et al. 2003). Eine kritische Diskussion von Modellen der Technikakzeptanz hinsichtlich ihrer Erklärungskraft für Senioren/innen geben Misoch et al. (2016). Das Almere-Modell bezieht sich spezifisch auf Assistive Roboter für ältere Erwachsene (Heerink et al. 2010) und berücksichtigt auch wahrgenommene Anpassungsfähigkeit und soziale Präsenz. Eine Erweiterung erfuhr das Modell im Kontext von Servicerobotern im Service Robot Acceptance Model (sRAM) (Wirtz et al. 2018).

Unter Berücksichtigung relevanter Technikakzeptanzmodelle und Skalen erstellte das IAF im Rahmen der bereits genannten Online-Studie einen Fragebogen, in welchem 13 verschiedene Faktoren erfragt wurden, welche die Roboterakzeptanz beeinflussen (Lehmann et al. 2020b). Basierend auf 13 Faktoren (generelle Einstellung gegenüber Robotern; negative Emotionen; positive Emotionen; Selbstwirksamkeit; Vertrauen; Nützlichkeit; Aufwand; Freude; Sicherheit; Kontrolle; Benutzerfreundlichkeit; Einstellung des sozialen Netzwerks; soziale Unterstützung) wurde ein Akzeptanzwert aus 21 einzelnen Variablen berechnet. Der Fragebogen wurde an 61 Personen einem Pretest unterzogen. Um die interne Konsistenz zu bestimmen wurde Cronbach's Alpha berechnet, diese war mit $>0,9$ hoch. Es zeigte sich, dass die Akzeptanz positiv mit der Nutzungsintention zusammenhängt $(\mathrm{r}=0,857, p<0,000)$. Besonders hohe Zustimmungen von den Befragten erhielten die beiden Fragen des Faktors Selbstwirksamkeit (,Nach einer entsprechenden Einweisung wäre ich in der Lage, einen Roboter zu nutzen“, 86,0\% stimmten eher bis vollkommen zu und „Ich hätte alle Fähigkeiten, um mit einem Roboter umzugehen“, 83,1\% stimmten eher bis vollkommen zu) und die Frage des Faktors zur sozialen Unterstützung („Ich denke, dass im Falle eines Heimaufenthaltes mir das Pflegepersonal beim Gebrauch eines Roboters helfen würde“, 87,3\% stimmten eher bis vollkommen zu).

\subsection{Emotionen gegenüber Robotern}

Neben kognitiv evaluativen und sozialen Faktoren fanden in den Modellen die Emotionen der Nutzenden bislang wenig umfassende Beachtung. Ältere Personen verwenden Technik jedoch nicht nur aufgrund ihres Nutzens (Díaz et al. 2013), auch die Emotionen der Nutzenden spielen dabei eine wichtige Rolle. Gerade in Bezug auf die Interaktionen älterer Personen mit Robotern in verschiedenen Umgebungen zeigten sich auch Faktoren wie Freude und Spass an der Interaktion als sehr wichtig (Papadopoulos et al. 2020). Dafür untersuchten Papadopoulos et al. (2020) in ei- 
nem systematischen Review die Interaktion mit humanoiden Robotern. Sie konnten 12 Studien, jeweils ohne experimentelles Design, mit 420 Personen einschliessen, wovon 307 über 60 Jahre alt waren. Die Studien hatten meist keine Baseline-Erhebung, beinhalteten nur Selbstauskunfts-Daten und die Stichproben waren klein. Auch hier besteht das Problem, dass wenig evidenzbasierte Ergebnisse mit oft nur kleinen Stichproben vorliegen.

In einer simulierten ${ }^{2}$ Roboter-Studie des IAF wurden 142 Testpersonen $(\mathrm{M}=$ 73,2 Jahre, $\mathrm{SD}=6,1$, Altersrange 58-87; 54,2\% Frauen) zu ihren Emotionen jeweils in unterschiedlichen Situationen und mit unterschiedlichem Aussehen des Roboters befragt. Insgesamt wurden mehr positive als negative Emotionen bejaht. Zusätzlich zeigte sich, dass in einer Situation, in welcher ein Roboter einer älteren Dame ein Getränk bringt (,Servicesituation“), weniger negative Emotionen bejaht wurden als in einer Situation, in welcher ein Roboter eine liegende Person wäscht (,Pflegesituation“) (Ruf et al. 2020b). Unabhängig von der Situation wurden mehr positive Emotionen bejaht, je menschenähnlicher der Roboter aussah. Mit der gleichen Untersuchungsmethodik wurden 35 Bachelorstudierende $(M=22,4$ Jahre, $\mathrm{SD}=5,5$, Altersrange 19-51; 85,7\% Frauen) der Pflege zu ihren Emotionen befragt (Lehmann et al. 2020a). Verglichen mit den älteren Studienteilnehmenden nannten die Studierenden signifikant mehr negative Emotionen, die älteren Studienteilnehmenden signifikant mehr positive Emotionen gegenüber Robotern generell. Dieser Unterschied zeigte sich auch bezüglich der Situation, in der mit dem Roboter interagiert wird, und bezüglich des Aussehens des Roboters. Eine eher negative Einstellung von professionellen Pflegenden oder pflegenden Angehörigen im Vergleich zu den älteren Erwachsenen selbst berichten auch verschiedene andere Studien (Bedaf et al. 2018; Hebesberger et al. 2017). Bedaf et al. (2018) liessen dafür zehn ältere Erwachsene mit einem Roboter in einer Wohnumgebung interagieren und interviewten anschliessend die älteren Personen, informelle Pflegende $(N=7)$ und professionelle Pflegende $(N=11)$. Hebesberger et al. (2017) nutzen Beobachtungsdaten, Interviews $(N=10)$ und Fragebogen $(N=70)$ mit dem Personal, um den Einsatz eines autonomen Roboters in einem Pflege-Krankenhaus in Österreich zu evaluieren.

\subsection{Bevorzugtes Aussehen eines Roboters}

Roboter werden von Anwender/innen auch aufgrund ihres Aussehens beurteilt, welches dazu beitragen kann, positive Gefühle gegenüber dem Roboter auszulösen und damit eine höhere Akzeptanz zu bewirken (Hwang et al. 2013). Die äussere Gestalt eines Roboters ist ein wesentlicher Faktor, da sie die Erwartungen beeinflusst, die Menschen bei der Interaktion mit einem System haben, und kann in unterschiedliche Kategorien, wie zum Beispiel zoomorph (tierähnlich) oder anthropomorph (menschenähnlich) eingeteilt werden (Miller und Feil-Seifer 2017). Sozial Assistive

\footnotetext{
2 Gemäss Broadbent (2017) lässt sich die Forschung zu Robotern in so genannte „,simulierte Roboterstudien“ und „reale Roboterstudien“ unterteilen (S. 637). Simulierte Studien verwenden dabei anstelle von realen Robotern schriftliche Beschreibungen, Fotos oder Videos von Robotern. Beide Studienarten können zum Wissen über die Mensch-Roboter-Beziehungen beitragen und bringen jeweils spezifische Vor- und Nachteile mit sich.
} 
Roboter, welche menschenähnliche Merkmale haben, fördern eher die Akzeptanz und Nutzung (Khosla et al. 2017). Hinsichtlich des Aussehens eines Roboters ist die „Uncanny Valley“ Hypothese (Mori 1970) viel diskutiert, welche besagt, dass je menschenähnlicher das Aussehen des Roboters, desto akzeptierter dieser ist, jedoch gibt es ab einem bestimmten Punkt der zu grossen Menschenähnlichkeit einen abrupten Abfall der Akzeptanz. Daher werden androide Roboter als unheimlich empfunden, deren Bewegungen zu mechanisch sind und dementsprechend nicht zu einem menschlichen Aussehen passen (Mara und Appel 2015). Dies verdeutlicht, dass das Aussehen nicht losgelöst von der Situation betrachtet werden kann, sondern es zum jeweiligen Einsatzfeld beziehungsweise der Aufgabe/Tätigkeit des Roboters passen muss (Broadbent 2017; Robinson et al. 2014). Robotern mit menschenähnlicher Gestalt werden eine Persönlichkeit, Emotionen und Intentionen zugeschrieben (Janowski et al. 2018), dabei führt die potenzielle Vermenschlichung dazu, dass die Roboter als ,,vertrauenswürdig“ erachtet werden. Darüber hinaus ist es wünschenswert, dass Gestalt und Fähigkeiten eines Roboters kongruent sind. Weitere Untersuchungen, wie das Review von Broadbent et al. (2009) zeigen, dass ältere Menschen diskrete, kleine Roboter sowie menschen- oder haustierähnliche gegenüber großen humanoiden Robotern bevorzugen (Torta et al. 2014; Wu et al. 2012). Torta et al. (2014) führten dafür eine Kurzzeit- und Langzeit-Evaluation eines kleinen Roboters bei älteren Personen $(N=8, M=77$ Jahre, Altersrange 70-95) in Österreich durch. $\mathrm{Wu}$ et al. (2012) führten drei Fokusgruppen mit 15 älteren Personen (Altersrange 66-89 Jahre; 12 Frauen; 7 litten an milden kognitiven Einschränkungen) durch.

Unter Vorgabe verschiedener Kategorien befragte das IAF 189 Personen, die im Durchschnitt 65,4 Jahre alt waren (von 17 bis 96; 57,6\% Frauen), in einer OnlineBefragung, welches Aussehen sie für einen Roboter bevorzugen würden (Lehmann et al. 2020b). Dabei zeigte sich ein heterogenes Bild: ein Viertel der Befragten wünschte sich ein Aussehen wie eine Maschine, es bevorzugte aber auch je über ein Fünftel ein menschliches Aussehen oder gab keine Präferenz für das Aussehen an. Weniger bevorzugt wurden die Kategorien Gebrauchsgegenstand, Fantasiewesen oder Tier. Männer bevorzugten etwas häufiger ein maschinelles Aussehen oder hatten keine Präferenz, während Frauen das menschliche und das maschinelle Aussehen bevorzugten.

\section{Einsatzbereiche von Robotern zur Unterstützung älterer Personen}

Roboter können zur Unterstützung im Alter sowohl im Privathaushalt als auch in Alters- und Pflegeheimen eingesetzt werden. Diese Einsatzorte konnten sich auch die 189 online befragten Personen $(M=65,4$ Jahre, $S D=16,6)$ aus der Deutschschweiz gut vorstellen (Lehmann et al. 2020b). Dabei wurde der Einsatz eines Roboters bei verschiedenen Aufgaben unterschiedlich akzeptiert. Solch unterschiedliche Präferenzen zeigten sich auch bei einer Fragebogen-Erhebung auf einer Messe in Genf ( $N=240,6 \%$ über 65 Jahre) von Ray et al. (2008), wobei die Übernahme von wiederkehrenden Reinigungstätigkeiten durch einen Roboter (wie zum Beispiel Staubsaugen oder Fensterputzen) eher gewünscht war als Tätigkeiten, die eher als persönlich wahrgenommen wurden (zum Beispiel Kochen). 


\subsection{Privathaushalt}

Beim Einsatz von Robotern im Privathaushalt steht der Erhalt der Selbständigkeit, Autonomie und Wohlbefinden im Zentrum (Janowski et al. 2018). Gerade mit der Möglichkeit länger in der eigenen Häuslichkeit zu verbleiben, konnten sich in einer repräsentativen telefonischen Befragung die Personen $(N=1003)$ die Nutzung eines Serviceroboters vorstellen (forsa 2016). Im Folgenden wird als Beispiel der Einsatz eines humanoiden Roboters zur Gesundheitsförderung von älteren Personen zuhause vorgestellt (Ruf et al. 2020a), da regelmässige Bewegung ein Schutzfaktor ist und sich eine stabile Nutzenevidenz für Gesundheit und Selbständigkeit im Alter zeigt (Büla et al. 2014; Woll und Servay 2013). Einige Studien weisen auf ein grosses Potenzial von humanoiden Robotern als Instruktoren und Motivatoren hin, die Umsetzbarkeit im häuslichen Kontext wurde jedoch zum ersten Mal von Ruf et al. (2020a) in dieser Art untersucht. Wie von Chi (2009) und Lehmann et al. (2019) gefordert, sollte mit Endnutzern/innen in Living Labs bzw. ihrer gewohnten Lebensumwelt zusammengearbeitet werden. Unter Living Lab wird dabei die natürliche Lebensumwelt der Endnutzenden verstanden, wo Forschende technische Systeme einsetzen, welche über längere Zeit hinweg in der realen Umgebung erprobt werden (Lehmann et al. 2019). Im Rahmen der Untersuchung trainierten fünf Senioren und zwei Seniorinnen ( $M=74$ Jahre, $S D=5,63$, Altersrange 67-84) eine Woche lang selbständig zuhause mit einem humanoiden Roboter als Trainingscoach. Die körperlichen Übungen konnten mit dem humanoiden Roboter durchgeführt werden, das Aussehen wurde als angenehm empfunden und der Roboter grundsätzlich als motivierend erlebt. Die Senioren/innen berichteten aber auch Hemmnisse. So wurden die Instruktionen des Roboters als zu unflexibel und die Unmöglichkeit den Übungsablauf zu variieren als unkomfortabel erlebt. Weiter wurde berichtet, dass sich bei immer gleichen Instruktionen ein Gewöhnungseffekt einstellt. So wurde der humanoide Roboter von den Teilnehmenden akzeptiert, jedoch mit den aktuell vorhandenen Begrenzungen nicht für den Einsatz bei älteren Personen weiterempfohlen (Ruf et al. 2020a). Diese Studie konnte die Möglichkeiten für einen Robotereinsatz bei älteren Personen im Privathaushalt aufzeigen, aber auch verdeutlichen, welche Herausforderungen noch zu bewältigen sind, bevor ein nachhaltiger Einsatz realisiert werden kann.

\subsection{Alters- und Pflegeheim}

Roboter können auch in Alters- und Pflegeheimen eingesetzt werden, zum Beispiel als Begleiter für ältere Bewohnende oder als Unterstützung für das Pflegepersonal. Es existieren Belege für eine positive Wirkung der Roboter Robbe PARO bei Menschen mit Demenz aus unterschiedlichen Ländern (Abdi et al. 2018). PARO wurde ursprünglich für die Aktivierung von Menschen mit Demenz entwickelt und wird aktuell erfolgreich im institutionellen Setting eingesetzt. Aber nicht nur dementielle Erkrankungen, auch Einsamkeit der Bewohner/innen stellt eine der Herausforderungen für Alters- und Pflegeheime dar. So sind Einsamkeit und soziale Isolation ein häufiges Phänomen bei älteren Menschen, welches sich negativ auf die Gesundheit auswirkt (Losada et al. 2012). Daher wurde im Rahmen einer qualitativen Studie des 
IAF die Wirkung des Einsatzes der Roboter Robbe PARO bei kognitiv nicht eingeschränkten, aber einsamen oder sozial isolierten Personen im Alters- und Pflegeheim untersucht. Während des jeweils dreimonatigen Studienzeitraums im Jahr 2019 wurde PARO in drei Heimen bei sechs Bewohnerinnen über 65 Jahren eingesetzt. Die Testung im Feld wurde jeweils im Einzelsetting durch vier Aktivierungstherapeutinnen vorgenommen. Es wurden regelmässig leitfadengestützte Interviews mit den Aktivierungstherapeutinnen zu Erfahrungen mit dem Einsatz von PARO durchgeführt, auditiv aufgezeichnet, protokollarisch transkribiert und inhaltsanalytisch ausgewertet. Dabei zeigten sich positive Effekte, indem soziale Isolation durchbrochen, sozialer Kontakt aufgebaut und der Kommunikationsfluss verbessert werden konnte, aber es zeigten sich auch Gewöhnungseffekte.

Ein weiteres Beispiel für den Einsatz eines Roboters im Alters- und Pflegeheim ist die Unterstützung des Pflegepersonals. Um geeignete Einsatzmöglichkeiten eines Roboters als Unterstützung zu definieren, wurde im Rahmen eines laufenden Forschungsprojektes mit der Beteiligung des IAF der Tagesablauf von Pflegepersonen durch die Forschenden in der natürlichen Umgebung begleitet. Durch die Beobachtungen und Gespräche mit dem Pflegepersonal zeigte sich grosses Potenzial, die Pflegepersonen bei körperlich anstrengenden, pflegefernen und zeitintensiven Arbeiten $\mathrm{zu}$ entlasten und damit ihr körperliches und emotionales Wohlbefinden zu stärken. Es war ersichtlich, dass eine Entlastung im Sinne von Abnahme diverser Aufgaben (zum Beispiel Aufräumen der Gemeinschaftsräume, Materialabholung) oder Unterstützung der Pflegeperson (zum Beispiel bei der Dokumentation) denkbar wäre. Auch in anderen Forschungsarbeiten wird berichtet, dass eine Entlastung durch den Einsatz robotischer Hilfe von Pflegekräften bei Aktivitäten wie zeitaufwändigen Routinearbeiten (Dokumentation, Materialbestellung, Medikamentenportionierung), bei Transport von Material und Personen, körperlich anstrengenden Arbeiten (Heben und Tragen) sowie kognitiv fordernden Arbeiten (Merken und Organisieren vieler und ungeplanter Aufgaben) gewünscht wird (Becker 2018; Graf et al. 2013). Um diesem Bedürfnis und Wunsch Rechnung zu tragen, wird im Rahmen des erwähnten laufenden Forschungsprojektes mit der Beteiligung des IAF gemeinsam mit einem Roboter-Entwickler und einem innovationsorientierten Alters- und Pflegeheim ein Roboter entwickelt, der spezifisch auf ihre Anforderungen zugeschnitten ist. Dies unter der Berücksichtigung der menschlichen, wirtschaftlichen, technischen und rechtlichen Sichtweise (SRF 2020).

\section{Grenzen des Robotereinsatzes zur Unterstützung im Alter}

Die durchgeführten Forschungsprojekte zeigen deutlich, dass es vielversprechende Möglichkeiten, aber auch Grenzen für den Einsatz von Robotern zur Unterstützung im Alter gibt. Neben aktuell noch vorhandenen technischen Schwierigkeiten und teilweise negativen Einstellungen von Senioren/innen und Professionellen, sind Barrieren vor allem in Bereichen der Umwelt, der Organisation, des gesellschaftlichen und kulturellen Milieus, der Politik und dem rechtlichen Rahmen zu sehen (Papadopoulos et al. 2020). 
Aus Sicht des IAF muss der Fokus auf der Sicht der Nutzenden liegen (sowohl der Senioren/innen, der Angehörigen als auch des Pflegepersonals), deren geäusserten Wünschen und Bedenken. Weiter sollten auch gesamtgesellschaftliche, wirtschaftliche und ethische Überlegungen in Betracht gezogen werden. Im Folgenden werden die Grenzen eines Robotereinsatzes bei älteren Menschen hinsichtlich technischer Machbarkeit, Einsatzfeldern, Funktionen und ethischen Bedenken aus der Sicht der Nutzenden dargestellt.

\subsection{Technische Machbarkeit}

Viele Nutzende haben aktuell ein oft utopisches Bild eines Roboters im Kopf, das nicht zuletzt aus Filmen resultiert (Teo 2020) und nicht mit den tatsächlichen Möglichkeiten der heutigen Robotersysteme übereinstimmt. Dadurch werden übersteigerte Erwartungen an die entwickelten Roboter herangetragen, die dann zwangsläufig enttäuscht werden und in einer geringen Akzeptanz resultieren können. Daher sind beim partizipativen Einbezug der Nutzenden die technischen Möglichkeiten nicht zu vernachlässigen, um einerseits gewünschte, aber andererseits auch realistische Roboter entwickeln zu können. So zeigte sich in einem Prototypen-Workshop des IAF, dass Roboter oft noch nicht so ausgereift sind, wie erwartet und gewünscht und daher eine realistische Einschätzung des konkreten Nutzens für sich selbst für die älteren Personen sehr schwer ist. Am Workshop nahmen 12 Senioren/innen (neun Männer und drei Frauen) von 70 bis 84 Jahre (im Durchschnitt 75 Jahre alt) teil. Sie sollten einen Robotergreifarm, der auf einer Plattform befestigt war, beurteilen. Der Prototyp lag lediglich in Form von schriftlichen Beschreibungen und Visualisierungen vor. Die anwesenden Teilnehmer/innen hatten vielfältige Anregungen, welche Funktionen dieser Arm übernehmen können sollte und wünschten sich dementsprechend weitere Attribute: so sollte der Roboterarm mobil sein und nicht nur fest an einem Platz stehen können, er sollte zwei Arme besitzen, um beispielsweise Getränke öffnen zu können, und er sollte eine grössere Tragfähigkeit haben, um Gegenstände über 0,5 kg heben zu können. Diese Ergebnisse zeigen Schwierigkeiten und Herausforderungen, die im Zusammenhang mit der technischen Machbarkeit auftreten. Da zurzeit viele Roboter erst als Prototypen bestehen und es sich um einen sehr dynamischen Markt handelt, wäre eine Aussage darüber, was nun die „besten“ Roboter sind, aktuell nicht vertretbar. Zumal die robotischen Systeme verschiedene Aufgaben im Altersbereich für unterschiedliche Nutzergruppen unterschiedlich gut erfüllen.

Auch beim Einsatz eines humanoiden Roboters zur Bewegungsaktivierung im häuslichen Kontext traten einige technische Schwierigkeiten auf (siehe Abschn. 4.1, Ruf et al. 2020a). Der Roboter benötigte zu lange, um betriebsbereit zu sein, reagierte nicht sofort auf Befehle, erkannte die ältere Person nicht oder fiel während der Bewegungsübungen um. Auch akustische Probleme traten auf, so dass die Computerstimme nicht gut verstanden oder als anstrengend wahrgenommen wurde. Allgemein machte der Roboter zu viele und zu lange Pausen, bei welchen den Nutzenden nicht klar war, ob sie gewollt waren oder der Roboter nicht funktionierte. 


\subsection{Einsatzfelder, Personenkreis, Funktionen}

Es zeigen sich grosse Unterschiede in gewünschten Einsatzfeldern, Funktionen und Personenkreis des Einsatzes von Robotern für ältere Personen, wenn potenzielle Nutzer/innen befragt werden. So waren in der bereits erwähnten Online-Befragung in der Deutschschweiz $(N=189)$ verschiedene Funktionen unterschiedlich gut vorstellbar für die Endnutzenden (Lehmann et al. 2020b). Für die meisten Befragten nicht vorstellbar war eine Konversation mit einem Roboter (72,2\%), pflegerische Tätigkeiten (zum Beispiel Waschen) durch einen Roboter $(69,0 \%)$, und der Roboter als Kommunikationshilfe zur Kommunikation mit Freunden und Verwandten $(58,3 \%)$. Auch der Personenkreis unterscheidet sich stark. So konnten sich die meisten befragten Personen einen Einsatz eines Roboters bei sich selbst oder fremden Personen vorstellen, jedoch nur 20,9\% bei den eigenen Eltern oder Grosseltern.

\subsection{Ethische Überlegungen}

Neben dem erhofften Nutzen und den Möglichkeiten eines Robotereinsatzes stellt sich die Frage, welche Funktionen und Einsatzbereiche von den Nutzenden gewünscht und vertretbar sind, und wo die Grenzen und Bedenken eines solchen Einsatzes liegen. So sind die betroffenen Personen im Altersbereich aufgrund kognitiver und/oder körperlicher Einschränkungen besonders vulnerabel und gleichzeitig auf besonders umfassende Unterstützung angewiesen. Bedenken hinsichtlich der Menschen mit Pflege- oder Assistenzbedarf sind unter anderem, dass sie durch den Einsatz robotischer System weniger soziale und emotionale Unterstützung erfahren und Einschränkungen in ihrer Privatsphäre und Handlungsfreiheit unterworfen werden, sowie getäuscht und infantilisiert werden könnten. Bedenken bezogen auf professionelle Pflegekräfte beziehen eine Veränderung ihrer Tätigkeit hin zu weniger beziehungsorientierter Pflege ein, sowie dass die bevorzugte Finanzierung robotischer Systeme zulasten von Verbesserungen im personellen Bereich (höhere Vergütung, geringere Arbeitsdichte, generelle Aufwertung des Pflegeberufs) geht (Deutscher Ethikrat 2020).

Die ethische Diskussion eines Robotereinsatz zur Unterstützung im Alter und seiner Bedeutung für die verschiedenen Nutzergruppen ist essenziell wichtig und wird aktiv und kontrovers geführt (Körtner 2016); einen umfassenden Überblick über die Debatte gibt das systematische Review von Vandemeulebroucke et al. (2018). Dabei soll, wie der Deutsche Ethikrat (2020) in seiner Stellungnahme betont, ,Robotertechnik grundsätzlich ein komplementäres und nicht ein substitutives Element der Pflege darstellen, welches immer in ein personales Beziehungsgeschehen eingebettet sein muss" (S. 13). Bei der Entwicklung und Anwendung von robotischen Systemen im Altersbereich ist die Berücksichtigung von ethischen Kernprinzipien erforderlich, welche sich auf die Prinzipien der biomedizinischen Ethik beziehen und Nichtschaden, Fürsorge, Selbstbestimmung, und Gerechtigkeit beinhalten (Beauchamp und Childress 2009; in Körtner 2016). Daneben werden wir durch die Digitalisierung mit zusätzlichen ethischen Fragen konfrontiert, die systematisch identifiziert und bedacht werden müssen. Dabei übersetzt die Digitale Ethik bestehende ethische Maßstäbe für eine digital geprägte Gesellschaft (BVDW 2019). Der Einsatz von 
Robotern im Altersbereich beinhaltet auch die Möglichkeit Daten aufzunehmen und zu speichern, was besondere ethische Fragestellungen für alle Nutzergruppen aufwirft. Es dürfen nicht mehr Daten als unbedingt nötig erfasst werden, weder von den Unterstützungs- respektive Pflegebedürftigen noch von den Betreuungs- respektive Pflegepersonen. Da diese Daten besonders sensibel sind, müssen sie ausreichend vor unberechtigtem Zugriff geschützt werden. Durch den Einsatz von künstlicher Intelligenz wird es zunehmend wichtiger, dass ethische Überlegungen auch bereits bei der Programmierung von Robotern mit bedacht werden. Die Europäische Gruppe für Ethik der Naturwissenschaften und der Neuen Technologien (EGE 2018) formuliert daher weitere ethische Grundsätze und demokratische Voraussetzungen wie Datenschutz und Privatsphäre und betonen, dass Roboter den Datenschutzvorschriften entsprechen müssen und keine Daten sammeln und verbreiten dürfen, für deren Nutzung und Verbreitung keine aufgeklärte Einwilligung vorliegt (S. 22). Gemäss Yew (2020) betreffen ethische Herausforderungen beim Einsatz von Robotern in der Pflege das Ausmass der Pflege durch Roboter, die Möglichkeit der Täuschung von vulnerablen Personen, (übermässiges) Vertrauen in und (übermässige) Bindung an Roboter, das Fehlen einer informierten Zustimmung und die mögliche Verletzung der Privatsphäre der Nutzenden. Das ethische Design eines Roboters bezieht sich auf den Prozess, durch den ethische Werte oder Prinzipien in der Entwicklung des Produktes eingearbeitet werden. Dafür muss ein grundlegender ethischer Rahmen vorhanden sein, der mögliche Handlungen und Entscheidungen eines Roboters von Anfang an bestimmt. Gleichzeitig soll ein Roboter aber auch durch die Interaktion mit seiner Umwelt ethische Werte dazu lernen. Zum Beispiel von der Europäischen Kommission (2020) gibt es inzwischen ethische Richtlinien, Normen und Vorschriften, die sich speziell auf die Konstruktion von Robotern und anderen künstlichen intelligenten Systemen beziehen.

Um ethische Vorbehalte und Bedenken bei der Nutzung technischer Assistenzsysteme zu identifizieren wurde im Rahmen der Studie „Ethische Fragen im Bereich Altersgerechter Assistenzsysteme“ das MEESTAR-Modell (,Modell zur ethischen Evaluation sozio-technischer Arrangements“) entwickelt, um in einen strukturierten Dialog treten zu können und anhand dessen ethische Fragestellungen zu systematisieren, zu analysieren, und potenzielle Chancen und zukünftige Handlungsfelder zu identifizieren (Manzeschke et al. 2013).

Bei der erwähnten Online-Befragung des IAF in der Deutschschweiz zeigte sich, dass ethische Bedenken vor allem den Verlust von persönlichen Kontakten oder Probleme mit dem Umgang mit sensiblen Daten betreffen. Fast die Hälfte der Befragten hat jedoch auch Bedenken, dass Arbeitskräfte ihren Job verlieren könnten oder die Privatsphäre nicht garantiert ist. Weitere Bedenken waren, dass die älteren Personen getäuscht, ihre Selbstbestimmung beeinträchtigt oder ihre Würde verletzt werden könnte (Lehmann et al. 2020b). Auch Ray et al. (2008) finden bei ihrer Fragebogen-Erhebung ( $N=240,6 \%$ über 65 Jahre) als negative Aspekte das Fehlen von zwischenmenschlichen Beziehungen. 


\section{Schlussfolgerung}

Der frühe Einbezug aller Endnutzenden ist notwendig, um die Akzeptanz einzelner robotischer Anwendungen sicherzustellen und Verbesserungsmöglichkeiten früh aufzeigen und damit einen erfolgreichen Einsatz des jeweiligen Roboters umsetzen zu können. Dabei ist darauf zu achten, alle Endnutzer/innen respektive Stakeholder zu berücksichtigen und miteinzubeziehen. So kann mitunter gerade die Sicht der Senioren/innen sich deutlich von der Sicht der Angehörigen oder dem professionellen Pflegepersonal unterscheiden.

Es zeigte sich, dass die Akzeptanz der gleichen Roboterlösung je nach Gruppe von Endnutzenden unterschiedlich ausfallen kann. Es sollte daher genau abgewogen werden, aufgrund welcher Einstellungen und Emotionen von welchen Nutzergruppen ein Robotereinsatz beurteilt wird. So sollte zwar auch das Pflegepersonal bei der Entwicklung technischer Lösungen partizipieren können, um die Entwicklung nach ihren Bedürfnissen zu gestalten, was bisher wenig erfolgte (Merda et al. 2017), jedoch müssen vor dem Einsatz eines Roboters im Dienstleitungs- und Pflegebereich auch die Einstellungen der älteren Personen berücksichtigt werden. Ein Einsatz sollte nicht nur aufgrund der Einstellung von jüngeren Personen abgelehnt werden. Ebenso muss die Entscheidung für den Einsatz eines Roboters zur Unterstützung im Alter in einer konkreten Situation jeweils individuell entschieden werden (Becker 2018).

Durch den Einbezug der Endnutzenden können auch konkrete Verbesserungsmöglichkeiten einer robotischen Lösung aufgezeigt werden. So konnten die sieben Senioren/innen, die mit einem humanoiden Roboter als Bewegungscoach trainierten, Verbesserungspotential aufzeigen, indem individuell adaptierbare und personalisierbare Lösungen, genug grosse Roboter, Erinnerungsfunktionen, Variationen der Kommunikation und Übungen, Ansagen von Pausen, Fehlerkorrekturen, Kompatibilität mit anderen Geräten und soziale Aspekte gewünscht wurden (Ruf et al. 2020a). Bei mehreren Einsatzbereichen unterschiedlicher Roboter (humanoider Roboter als Trainingscoach, Companion Roboter zur Verbesserung der sozialen Isolation) zeigten sich jedoch neben den positiven Effekten auch deutliche Gewöhnungseffekte. Dies lässt die Überlegung zu, ob der Einsatz jeweils zeitlich begrenzt sein sollte bei gewissen Anwendungen, wie dem Einsatz eines Companion Roboters. Wichtig ist dabei aber auch festzuhalten, dass es sich zurzeit (bis auf wenige Ausnahmen, zum Beispiel Studien mit der Roboterrobbe PARO) noch um kleine und eher explorative Studien handelt und insofern auf dieser Basis keine allgemeingültigen Aussagen getroffen werden können.

Selbstverständlich müssen in Zukunft auch Überlegungen zu finanziellen Aspekten gemacht werden, wofür eine systematische Analyse des praktischen Mehrwerts anzustreben ist, da bislang Nachweise des Nutzens oftmals fehlen (Merda et al. 2017). Im Bereich der ethischen, legalen und sozialen Aspekte (ELSA) bestehen aktuell noch viele offene Fragen, welche diskutiert werden müssen (siehe hierzu ausführlicher Bischof et al. 2018; Manzeschke et al. 2013). Wichtig ist, dass das Thema Robotereinsatz im Altersbereich nur interdisziplinär bearbeitet werden kann, um gemeinsam die beste Lösung für alle Nutzergruppen und die Gesellschaft zu erlangen. 
Funding Open access funding provided by University of Applied Sciences Eastern Switzerland.

Open Access Dieser Artikel wird unter der Creative Commons Namensnennung 4.0 International Lizenz veröffentlicht, welche die Nutzung, Vervielfältigung, Bearbeitung, Verbreitung und Wiedergabe in jeglichem Medium und Format erlaubt, sofern Sie den/die ursprünglichen Autor(en) und die Quelle ordnungsgemäß nennen, einen Link zur Creative Commons Lizenz beifügen und angeben, ob Änderungen vorgenommen wurden.

Die in diesem Artikel enthaltenen Bilder und sonstiges Drittmaterial unterliegen ebenfalls der genannten Creative Commons Lizenz, sofern sich aus der Abbildungslegende nichts anderes ergibt. Sofern das betreffende Material nicht unter der genannten Creative Commons Lizenz steht und die betreffende Handlung nicht nach gesetzlichen Vorschriften erlaubt ist, ist für die oben aufgeführten Weiterverwendungen des Materials die Einwilligung des jeweiligen Rechteinhabers einzuholen.

Weitere Details zur Lizenz entnehmen Sie bitte der Lizenzinformation auf http://creativecommons.org/ licenses/by/4.0/deed.de.

\section{Literatur}

Abdi J, Al-Hindawi A, Ng T, Vizcaychipi MP (2018) Scoping review on the use of socially assistive robot technology in elderly care. Bmj Open 8:e18815. https://doi.org/10.1136/bmjopen-2017-018815

Abowd GD, Bobick AF, Essa IA, Mynatt ED, Rogers WA (2002) The aware home: a living laboratory for technologies for successful aging. AAAI Technical Report WS-02-02

Agnihotri R, Gaur S (2016) Robotics: a new paradigm in geriatric healthcare. Gerontechnology 15:146-161. https://doi.org/10.4017/gt.2016.15.3.004.00

Arras KO, Cerqui D (2005) Do we want to share our lives and bodies with robots? A 2000-people survey. Technical report Nr. 0605-001. Autonomous Systems Lab, Swiss Federal Institute of Technology, EPFL, Lausanne

Beauchamp T, Childress JF (2009) Principles of biomedical ethics, 6. Aufl. Oxford University Press, Oxford

Becker H (2018) Robotik in der Gesundheitsversorgung: Hoffnungen, Befürchtungen und Akzeptanz aus Sicht der Nutzerinnen und Nutzer. In: Bendel O (Hrsg) Pflegeroboter. Springer Gabler, Wiesbaden, S 229-248

Becker H, Khiri N (2018) Digitalisierung in der Pflege - ein gedanklicher Zwischenhalt. A S P Schweiz Fachmag Gesundheitswes 2:28-32

Becker H, Scheermesser M, Früh M, Treusch Y, Auerbach H, Hüppi RA et al (2013) Robotik in Betreuung und Gesundheitsversorgung. TA-SWISS, Zentrum für Technologiefolgen-Abschätzung 58. https://www.research-collection.ethz.ch/bitstream/handle/20.500.11850/65166/eth-6329-01.pdf. Zugegriffen: 23. Juni 2020

Bedaf S, Marti P, Amirabdollahian F, de Witte L (2018) A multi-perspective evaluation of a service robot for seniors: the voice of different stakeholders. Disabil Rehabil Assist Technol 13:592-599. https:// doi.org/10.1080/17483107.2017.1358300

Bischof A, Herms R, Eibl M (2018) MERS-MRI - Methodische, ethische, rechtliche und soziale Implikationen für die Mensch-Roboter-Interaktion in Alltagswelten. In: Dachselt R, Weber G (Hrsg) Mensch und Computer 2018 - Workshopband. Gesellschaft für Informatik e. V.,, Bonn https://doi. org/10.18420/muc2018-ws14-0544

Bitkom Research (2018) Grosse Offenheit für digitale Helfer in der Pflege. https://www.bitkom.org/Presse/ Presseinformation/Grosse-Offenheit-fuer-digitale-Helfer-in-der-Pflege.html. Zugegriffen: 24. Sept. 2020

Broadbent E (2017) Interactions with robots: the truths we reveal about ourselves. Annu Rev Psychol 68:627-652. https://doi.org/10.1146/annurev-psych-010416-043958

Broadbent E, Kuo IH, Lee YI, Rabindran J, Kerse N, Stafford R, MacDonald BA (2010) Attitudes and reactions to a healthcare robot. Telemed J E Health 16:608-613. https://doi.org/10.1089/tmj.2009. 0171 
Broadbent E, Stafford R, MacDonald B (2009) Acceptance of healthcare robots for the older population: review and future directions. Int J Soc Robot 1:319-330. https://doi.org/10.1007/s12369-009-00306

Broekens J, Heerink M, Rosendal H (2009) Assistive social robots in elderly care: a review. Gerontechnology 8:94-103. https://doi.org/10.4017/gt.2009.08.02.002.00

Büla C, Jotterand S, Martin BW, Bize R, Lenoble-Hoskovec C, Seematter-Bagnoud L (2014) Bewegung im Alter: Dafür ist es nie zu spät! Swiss Med Forum 14:836-841

Bundesamt für Statistik (2019) Gesundheit im Alter. https:/www.bfs.admin.ch/bfs/de/home/statistiken/ gesundheit/gesundheitszustand/alter.html. Zugegriffen: 23. Juni 2020

Bundesamt für Statistik (2020a) Bevölkerungsentwicklung im Jahr 2019: provisorische Ergebnisse. Die Bevölkerung der Schweiz ist 2019 erneut gewachsen und gealtert. https://www.bfs.admin.ch/bfs/de/ home/statistiken/bevoelkerung.assetdetail.12247181.html. Zugegriffen: 23. Juni 2020

Bundesamt für Statistik (2020b) Künftige Bevölkerungsentwicklung. Szenarien zur Bevölkerungsentwicklung der Schweiz und der Kantone 2020-2050. Bundesamt für Statistik, Neuchâtel

BVDW Bundesverband Digitale Wirtschaft (BVDW) e. V. (2019) Mensch, Moral, Maschine. Digitale Ethik, Algorithmen und künstliche Intelligenz. https://www.bvdw.org/fileadmin/bvdw/upload/ dokumente/BVDW_Digitale_Ethik.pdf. Zugegriffen: 24. Sept. 2020

Chi EH (2009) A position paper on "living laboratories": rethinking ecological designs and experimentation in human-computer interaction. In: Jacko JA (Hrsg) Human-computer interaction. New trends. HCI 2009. Lecture Notes in Computer Science, Bd. 5610. Springer, Berlin, Heidelberg https://doi. org/10.1007/978-3-642-02574-7_67

Davis FD, Bagozzi RP, Warshaw PR (1989) User acceptance of computer technology: a comparison of two theoretical models. Manage Sci 35:982-1003. https://doi.org/10.1287/mnsc.35.8.982

Decker M (2010) Ein Abbild des Menschen: Humanoide Roboter. In: Bölker M, Gutmann M, Hesse W (Hrsg) Information und Menschenbild. Springer, Heidelberg, S 41-62

Deutscher Ethikrat (2020) Robotik für gute Pflege. Stellungnahme. https://www.ethikrat.org/fileadmin/ Publikationen/Stellungnahmen/deutsch/stellungnahme-robotik-fuer-gute-pflege.pdf. Zugegriffen: 23. Juni 2020

Díaz M, Saez-Pons J, Heerink M, Angulo C (2013) Emotional factors in robot-based assistive services for elderly at home. Proceedings of the 22nd IEEE International Symposium on Robot and Human Interactive Communication 711-716. https://upcommons.upc.edu/bitstream/handle/2117/20246/ThA1T1. 6.pdf. Zugegriffen: 23. Juni 2020

Europäische Gruppe für Ethik der Naturwissenschaften und der neuen Technologien (EGE) (2018) Erklärung zu künstlicher Intelligenz, Robotik und ,autonomen“ Systemen. https://ec.europa.eu/research/ ege/pdf/ege_ai_statement_2018_de.pdf. Zugegriffen: 24. Sept. 2020

European Commission (2017) Special Eurobarometer 460. Attitudes towards the impact of digitisation and automation on daily life. https://ec.europa.eu/jrc/communities/sites/jrccties/files/ebs_460_en.pdf. Zugegriffen: 23. Juni 2020

European Commission (2020) Ethics guidelines for trustworthy AI. https://ec.europa.eu/digital-singlemarket/en/news/ethics-guidelines-trustworthy-ai. Zugegriffen: 30. Sept. 2020

Forsa. Politik- und Sozialforschung GmbH (2016) „Service-Robotik: Mensch-Technik-Interaktion im Alltag“. Ergebnisse einer repräsentativen Befragung. Berlin, Deutschland. https://www.bmbf.de/files/ BMBF_forsa_Robotik_FINAL2016.pdf. Zugegriffen: 23. Juni 2020

Graf B, Heyer T, Klein B, Wallhoff F (2013) Servicerobotik für den demographischen Wandel. Mögliche Einsatzfelder und aktueller Entwicklungsstand. Bundesgesundheitsblatt Gesundheitsforschung Gesundheitsschutz 56:1145-1152

Hebesberger D, Koertner T, Gisinger C, Pripfl J (2017) A long-term autonomous robot at a care hospital: a mixed methods study on social acceptance and experiences of staff and older adults. Int J Soc Robot 9:417-429. https://doi.org/10.1007/s12369-016-0391-6

Hedtke-Becker A, Hoevels R, Otto U, Stumpp G, Beck S (2012) Zu Hause wohnen wollen bis zuletzt. In: Pohlmann S (Hrsg) Altern mit Zukunft. Springer, Wiesbaden, S 141-176

Heerink M, Kröse B, Evers V, Wieling B (2010) Assessing acceptance of assistive social agent technology by older adults: the Almere Model. Int J Soc Robot 2:361-375. https://doi.org/10.1007/s12369-0100068-5

Hudson J, Orviska M, Hunady J (2017) People's attitudes to robots in caring for the elderly. Int J of Soc Robotics 9:199-210 
Hülsken-Giesler M, Daxberger S (2018) Robotik in der Pflege aus pflegewissenschaftlicher Perspektive. In: Bendel O (Hrsg) Pflegeroboter. Springer, Berlin Heidelberg, S 125-139 https://doi.org/10.1007/ 978-3-658-22698-5_7

Hwang J, Park T, Hwang W (2013) The effects of overall robot shape on the emotions invoked in users and the perceived personalities of robot. Appl Ergon 44:459-471. https://doi.org/10.1016/j.apergo.2012. 10.010

International Federation of Robotics (2020) Service robots. https://ifr.org/service-robots/. Zugegriffen: 23. Juni 2020

Janowski K, Ritschel H, Lugrin B, André E (2018) Sozial interagierende Roboter in der Pflege. In: Bendel O (Hrsg) Pflegeroboter. Springer Gabler, Wiesbaden, S 63-87

Kehl C (2018) Robotik und assistive Neurotechnologien in der Pflege - gesellschaftliche Herausforderungen. TAB-Arbeitsbericht Nr. 177.. Büro für Technikfolgen-Abschätzung beim Deutschen Bundestag (TAB), Berlin

Khosla R, Nguyen K, Chu M-T (2017) Human robot engagement and acceptability in residential aged care. Int J Hum Comput Interact 33:510-522. https://doi.org/10.1080/10447318.2016.1275435

Körtner T (2016) Ethical challenges in the use of social service robots for elderly people. Z Gerontol Geriatr 49:303-307. https://doi.org/10.1007/s00391-016-1066-5

Lee HR, Sabanovic S, Chang W-L, Nagata S, Piatt J, Bennett C, Hakken D (2017) Steps toward participatory design of social robots: mutual learning with older adults with depression. 12th ACM/IEEE International Conference on Human-Robot Interaction, S 244-253 https://doi.org/10.1145/2909824. 3020237

Lehmann S, Hämmerle V, Pauli C, Misoch S (2019) Partizipative Testung assistiver Technik. Das Konzept der Living Labs 65+ des IKOA-FHS. In: Friedrich P, Fuchs D (Hrsg) 6. Ambient Medicine Forum. Assistive Technik für selbstbestimmtes Wohnen. Cuvillier, Göttingen, S 103-107

Lehmann S, Ruf E, Misoch S (2020b) Robot use for older adults—attitudes, wishes and concerns. First results from Switzerland. Proceedings of the 22nd International Conference on Human-Computer Interaction.

Lehmann S, Pauli C, Misoch S (2017) Partizipative Forschung auf höchster Stufe: Seniorinnen und Senioren beforschen ihre eigene Altersgruppe. eNewsletter Wegweiser Bürgergesellschaft 12/2017 vom 20.12.2017. https://www.buergergesellschaft.de/fileadmin/pdf/gastbeitrag_lehmann_etal_171220. pdf. Zugegriffen: 23. Juni 2020

Lehmann S, Ruf E, Misoch S (2020a) Emotions evoked by robots-comparison of older adults and students. Int J Mech Mechatronics Eng 14:8. https://publications.waset.org/abstracts/114688/emotionsevoked-by-robots-comparison-of-older-adults-and-students. Zugegriffen: 21. Okt. 2020

Losada A, Márquez-González M, García-Ortiz L, Gómez-Marcos MA, Fernández-Fernández V, Rodríguez-Sánchez E (2012) Loneliness and mental health in a representative sample of community-dwelling Spanish older adults. J Psychol 146:277-292. https://doi.org/10.1080/00223980.2011.582523

Manzeschke A, Weber K, Rother E, Fangerau H (2013) Ergebnisse der Studie „Ethische Fragen im Bereich Altersgerechter Assistenzsysteme“. VDI/VDE Innovation + Technik GmbH, Berlin (VDI/VDE). http://www.mtidw.de/grundsatzfragen/begleitforschung/dokumente/ethische-fragenim-bereichaltersgerechter-assistenzsysteme-1. Zugegriffen: 29. Sept. 2020

Mara M, Appel M (2015) Roboter im Gruselgraben: Warum uns menschenähnliche Maschinen oft unheimlich sind. In: In-Mind Magazin 5. Medienpsychologie Teil 2: Medien, Nachrichten und wir

Mayring P (2000) Qualitative Inhaltsanalyse. Forum Qualitative Sozialforschung / Forum: Qualitative Social Research 1:Art. 20. https://www.researchgate.net/publication/215666096_Qualitative_Content_ Analysis. Zugegriffen: 23. Juni 2020

Mercay C, Grünig A (2016) Gesundheitspersonal in der Schweiz - Zukünftiger Bedarf bis 2030 und die Folgen für den Nachwuchsbedarf (Obsan Bulletin 12/2016). Schweizerisches Gesundheitsobservatorium, Neuchâtel

Merda M, Schmidt K, Kähler B (2017) Pflege 4.0 - Einsatz moderner Technologien aus der Sicht professionell Pflegender. Forschungsbericht. Bonifatius GmbH, Paderborn. https://www.bgw-online. de/SharedDocs/Downloads/DE/Medientypen/BGW\%20Broschueren/BGW09-14-002-Pflege-4-0Einsatz-moderner-Technologien_Download.pdf?_blob=publicationFile. Zugegriffen: 23. Juni 2020

Miller B, Feil-Seifer D (2017) Embodiment, situatedness, and morphology for humanoid robots interacting with people. In: Goswami A, Vadakkepat P (Hrsg) Humanoid robotics: a reference. Springer, Dordrecht, S 1-23 https://doi.org/10.1007/978-94-007-7194-9_130-1 
Misoch S, Pauli C, Ruf E (2016) Technikakzeptanzmodelle: Theorieübersicht und kritische Würdigung mit Fokus auf ältere Nutzer/innen (60+). In: Weidner R (Hrsg) Technische Unterstützungssysteme, die die Menschen wirklich wollen. Konferenzband. Helmut-Schmidt-Universität, Hamburg, S 107-115

Mori M (1970) The uncanny valley. Energy 7:33-35 (https://spectrum.ieee.org/automaton/robotics/ humanoids/the-uncanny-valley)

Nitto H, Taniyama D, Inagaki H (2017) Social acceptance and impact of robots and artificial intelligence. Findings of survey in Japan, the U.S. and Germany. Nomura Research Institute (NRI Papers, 211). https://www.nri.com/-/media/Corporate/en/Files/PDF/knowledge/report/cc/papers/2017/np2017211. pdf?la=en\&hash=A730998FD55F6D58DF95F3479E3B709FC8EF83F4. Zugegriffen: 23. Juni 2020

Papadopoulos I, Koulouglioti C, Lazzarino R, Ali S (2020) Enablers and barriers to the implementation of socially assistive humanoid robots in health and social care: a systematic review. BMJ Open 10:e33096. https://doi.org/10.1136/bmjopen-2019-033096

Pino M, Boulay M, Jouen F, Rigaud AS (2015) “Are we ready for robots that care for us?" Attitudes and opinions of older adults toward socially assistive robots. Front Aging Neurosci 7:141. https://doi.org/ 10.3389/fnagi.2015.00141

Ray C, Mondada F, Siegwart R (2008) What do people expect from robots? Proceedings of the IEEE/RSJ International Conference on Intelligent Robots and Systems (IROS'08), S 3816-3821

Robinson H, MacDonald B, Broadbent E (2014) The role of healthcare robots for older people at home: a review. Int J Soc Robot 6:575-591. https://doi.org/10.1007/s12369-014-0242-2

Ruf E, Lehmann S, Misoch S (2020a) Motivating older adults to exercise at home: suitability of a humanoid robot. In: Guldemond N, Ziefle M, Maciaszek L (Hrsg) Proceedings of the 6th international conference on information and communication technologies for ageing well and e-health, S 113-120 https://doi.org/10.5220/0009341001130120

Ruf E, Lehmann S, Misoch S (2020b) Service robots: emotions of older adults in different situations. In: Guldemond N, Ziefle M, Maciaszek L (Hrsg) Proceedings of the 6th international conference on information and communication technologies for ageing well and e-health, S 15-25 https://doi.org/ $10.5220 / 0009324500150025$

Seifert A, Ackermann T (2020) Digitalisierung und Technikeinsatz in Institutionen für Menschen im Alter. Zentrum für Gerontologie, Zürich (Studie im Auftrag von CURAVIVA Schweiz)

Sixsmith A, Gutmann G (2013) Technologies for active aging Bd. 9. Springer, New York

SRF (2020) Künstliche Intelligenz. Wie soziale Roboter in Altersheimen helfen sollen. https://www.srf. ch/news/schweiz/kuenstliche-intelligenz-wie-soziale-roboter-in-altersheimen-helfen-sollen. Zugegriffen: 29. Sept. 2020

Teo Y (2020) Recognition, collaboration and community: science fiction representations of robot carers. Robot \& Frank, Big Hero 6 and Humans. Med Humanities. https://doi.org/10.1136/medhum-2019011744

Torta E, Werner F, Johnson DO, Juola JF, Cuijpers RH, Bazzani M, Oberzaucher J, Lemberger J, Lewy H, Bregman J (2014) Evaluation of a small socially-assistive humanoid robot in intelligent homes for the care of the elderly. J Intell Robot Syst 76:57-71. https://doi.org/10.1007/s10846-013-0019-0

Vandemeulebroucke T, Dierckx de Casterlé B, Gastmans C (2018) The use of care robots in aged care: a systematic review of argument-based ethics literature. Arch Gerontol Geriatr 74:15-25. https://doi. org/10.1016/j.archger.2017.08.014

Vaupel J (2000) Setting the stage: a generation of centenarians? Wash Q 23:197-200. https://doi.org/10. $1162 / 016366000561097$

Venkatesh V, Bala H (2008) Technology acceptance model 3 and a research agenda on interventions. Decis Sci 39:273-315. https://doi.org/10.1111/j.1540-5915.2008.00192.x

Venkatesh V, Davis FD (2000) A theoretical extension of the technology acceptance model: four longitudinal field studies. Manage Sci 46:186-204. https://doi.org/10.1287/mnsc.46.2.186.11926

Venkatesh V, Morris MG, Davis GB, Davis FD (2003) User acceptance of information technology: toward a unified view. MIS Q 27:425-478. https://doi.org/10.2307/30036540

Wirtz J, Patterso PG, Kunz WH, Gruber T, Lu VN, Paluch S, Martins A (2018) Brave new world: service robots in the frontline. J Serv Manag 29:907-931. https://doi.org/10.1108/JOSM-04-201-0119

Woll A, Servay S (2013) Körperliche Aktivität und Gesundheit im Alter. Public Health Forum 21:10-12. https://doi.org/10.1016/j.phf.2013.03.021

World Health Organization (2015) World report on ageing and health. World Health Organization, Geneva

Wu Y-H, Wrobel J, Cornuet M, Kerhervé H, Damnée S, Rigaud A-S (2014) Acceptance of an assistive robot in older adults: a mixed-method study of human-robot interaction over a I-month period in the Living Lab setting. Clin Interv Aging 9:801-811. https://doi.org/10.2147/CIA.S56435 
Wu YH, Fassert C, Rigaud AS (2012) Designing robots for the elderly: appearance issue and beyond. Arch Gerontol Geriatr 54:121-126. https://doi.org/10.1016/j.archger.2011.02.003

Yew GCK (2020) Trust in and ethical design of carebots: the case for ethics of care. Int J of Soc Robotics. https://doi.org/10.1007/s12369-020-00653-w

Zentrum für Qualität in der Pflege (2019) ZQP-Report. Pflege und digitale Technik. Zentrum für Qualität in der Pflege, Berlin (https://www.zqp.de/digitalisierung-pflege/)

Zöllick JC, Kuhlmey A, Suhr R, Eggert S, Nordheim J, Blüher S (2020) Akzeptanz von Technikeinsatz in der Pflege. In: Jacobs K, Kuhlmey A, Greß S, Klauber J, Schwinger A (Hrsg) Pflege-Report 2019. Springer, Berlin, Heidelberg, S 211-218 https://doi.org/10.1007/978-3-662-58935-9_17 